\title{
A hybrid model of acute unscheduled cancer care provided by a hospital-based acute care clinic and the emergency department: a descriptive study
}

\author{
Jason J. Bischof ${ }^{1,2}$ (1) $\cdot$ Montika Bush ${ }^{1} \cdot$ Rayad Bin Shams ${ }^{1}$. Frances A. Collichio ${ }^{3}$. Timothy F. Platts-Mills ${ }^{1,4}$
}

Received: 19 October 2020 / Accepted: 30 May 2021 / Published online: 5 June 2021

(c) The Author(s), under exclusive licence to Springer-Verlag GmbH Germany, part of Springer Nature 2021

\begin{abstract}
Purpose Patients with cancer often experience medical events that require immediate evaluation. These evaluations typically occur in an emergency department (ED), but there is increasing interest in providing this care in other settings. We report on a novel care model whereby a nursing hotline is used to triage patients to the ED or to the North Carolina Cancer Hospital Infusion Center (NCCHIC).

Methods A retrospective study of adult patients with a neoplasm diagnosis seeking acute care at a large academic hospital pre- and post-initiation of the novel care model in January of 2016. Patients were identified by querying the electronic medical record and clinic administrative data during matched 6 month pre- and post-periods.

Results During the pre-initiation period, 1346 patients visited the ED on 1651 occasions ( $76.1 \%$ admission rate). In the post-initiation period, 1434 patients visited the ED on 1797 occasions ( $81.5 \%$ admission rate), and 246 patients visited the NCCHIC on 322 occasions (68.9\% admission rate). The emergency severity index (ESI) in the pre-initiation ED group was primarily ESI 2 (30.6\%) and ESI 3 (65.4\%). In the post-initiation ED group, the ESI was similar (32.6\% ESI 2 and $64.2 \%$ ESI 3). In contrast, the NCCHIC predominantly treated lower acuity patients (65.8\% calculated ESI of $4 / 5)$.

Conclusions This model demonstrates a multidisciplinary partnership to providing acute unscheduled care for patients with cancer. In the early implementation phase of this model, approximately $15 \%$ of patients, generally of lower acuity, were seen in the NCCHIC.
\end{abstract}

Keywords Acute care $\cdot$ Emergency oncology $\cdot$ Ambulatory care $\cdot$ Unscheduled care

\section{Introduction}

Between 2006 and 2012, 29.5 million emergency department (ED) visits were made by patients with cancer and visit numbers increased year over year [1]. These patients frequently experience acute symptoms such as fever and shortness of

Jason J. Bischof

jason.bischof@osumc.edu

1 Department of Emergency Medicine, University of North Carolina School of Medicine, Chapel Hill, NC, USA

2 Department of Emergency Medicine, The Ohio State University Wexner Medical Center, 760 Prior Hall, 376 W. 10th Avenue, Columbus, OH 43210, USA

3 Lineberger Comprehensive Cancer Center, University of North Carolina At Chapel Hill, Chapel Hill, NC, USA

4 Quantworks, Inc., Carrboro, NC, USA breath which require rapid evaluation and treatment. Many of these presentations can be due to life-threatening conditions requiring emergent stabilization leading many patients to be referred to the ED setting by outpatient providers or by self-referral. However, in addition to life-threatening conditions, many patients present for unscheduled care due to uncontrolled symptoms such as pain and nausea [2]. A portion of these presentations can potentially be treated in non-ED settings such as an infusion or acute care clinic.

The increased acute care needs of patients with cancer and the increased specialization and complexity of cancer care are leading to an increased focus on acute oncology that requires a multifaceted approach to care provision $[3,4]$. New models of care to provide unscheduled care to patients with cancer are being developed with pilot studies demonstrating the potential benefit of multidisciplinary care for symptom management $[3,5-10]$. 
In an effort to improve the care provided to North Carolina's patient population with cancer, the University of North Carolina Cancer Hospital and the University of North Carolina ED implemented a novel care model in 2016. A hybrid care model was developed where cancer patients were encouraged to contact a nursing hotline to discuss their current symptoms and are then triaged either to the ED or to the North Carolina Cancer Hospital Infusion Center (NCCHIC) for further evaluation during business hours. The purpose of this study is to characterize and describe the care of patients with cancer receiving unscheduled acute care through this novel multidisciplinary partnership between the ED and NCCHIC.

\section{Methodology}

This retrospective study evaluates the transition to a novel acute care model for patients with cancer seen at an academic medical center affiliated with a comprehensive cancer center. The NCCHIC is located on the 3rd floor of the institution's cancer hospital which is physically contiguous with the rest of the medical center which includes the ED. The NCCHIC testing capabilities include full laboratory services, basic portable $\mathrm{x}$-ray imaging, and the availability of on-call supervising oncologist. Study data was collected via chart review during 6-month matched comparison periods before and after initiation of NCCHIC operations in January of 2016. The pre-intervention period was from July 1 st 2015 to December 31st 2015. The after-intervention period was from July 1st 2016 to December 31st 2016. The selected preand post-initiation periods cover the same calendar months to reduce confounding due to seasonal variations affecting patient presentations (e.g., influenza season). We excluded the 6 months immediately following the start-up date of the NCCHIC (washout period) to allow for NCCHIC protocol development and familiarization with this new care pathway by patients and providers. Patients aged 18 years or older with a previously diagnosed neoplasm (International Classification of Diseases-10: C00-D49) documented in their electronic medical chart presenting for acute unscheduled care in the ED or NCCHIC during the two study periods were included. Patients under the age of 18 were excluded as these patients are not eligible for care in the NCCHIC.

In addition to the telephonic triage referrals, patients could be referred to the NCCHIC directly from their outpatient oncologists' offices or walk-in to the NCCHIC for triage without referral. For patients presenting as walk-ins, the patient's primary oncologist and the patient's appropriateness for NCCHIC treatment was determined in conjunction with clinic staff. Patients presenting to the NCCHIC with symptoms concerning for myocardial infarction or acute stroke were referred directly to the ED due to the concern for acute life-threatening causes. Care was provided in the NCCHIC by two Board Certified Adult-Gerontology Nurse Practitioners who cross cover the cancer hospital's infusion clinic and on an as needed basis in collaboration with supervising on-call oncologists had the ability to admit patients directly from the NCCHIC to an oncology service without a need for transfer to the ED. These two advance practitioner providers triaged all of the calls based on established triage guidelines (Table 1) and their clinical judgement. Admitted patients from the NCCHIC who were pending bed placement at the time of the clinic's closing were transferred to the ED for continued care while awaiting hospitalization.

Basic demographics and clinical characteristics were obtained for all ED visits from the electronic medical record. Data for all NCCHIC visits were obtained from clinic administrative data if available and by chart review by trained study staff. If multiple different types of malignancy were noted in a single patient chart, each entry was recorded. Among ED patients, the chief complaint or reason for presentation was coded in a manner adapted from a previously published classification system from a similar population by a board certified emergency physician [11]. No such information was available for NCCHIC visits and as a result the final diagnosis was substituted for chief complaint and classified similarly. The emergency severity index (ESI) was obtained from the medical record for all ED patients. ESI was not available in the NCCHIC; as a result, it was calculated for NCCHIC patient visits via information obtained by chart review $[12,13]$.

All data was managed in REDCap and descriptive statistics were computed from de-identified datasets using STATA 15 (StataCorp, College Station, TX) [14]. The local institutional review board approved this study.

\section{Results}

During the study period, a total of 3770 patient encounters were identified as meeting inclusion criteria. In the pre-initiation period, 1346 patients visited the ED on 1651 occasions (76.1\% admission rate). In the post-initiation period, 1434 patients visited the ED on 1797 occasions (81.2\% admission rate), and another 246 patients visited the NCCHIC on 322 occasions (68.9\% admission rate).

Patient characteristics are reported in Table 2 with a notable prevalence of patients with central nervous system (CNS), gastrointestinal, head and neck, lung, and skin malignancies in the ED group. A higher proportion of patients presented to the NCCHIC with bladder, renal, and breast malignancy than to the ED.

Encounter characteristics are reported in Table 3. Notably, the ESI in the pre-initiation group was primarily ESI 2 (30.6\%) and ESI 3 (65.4\%). ESI in the ED post-initiation 
Table 1 Telephonic triage protocol for the North Carolina Cancer Hospital Infusion Center (NCCHIC)

\begin{tabular}{|c|c|}
\hline Appropriate for NCCHIC evaluation & Excluded from NCCHIC evaluation \\
\hline $\begin{array}{l}\text { - Currently receiving treatment with oncologist in the last } 3 \text { months } \\
\text { - Fever }>100.5 \mathrm{~F} \\
\text { - Shaking or chills } \\
\text { - Increasing shortness of breath with activity } \\
\text { - New fatigue affecting performance status } \\
\text { - Redness, swelling, drainage or pain at a catheter site } \\
\text { - Dehydration } \\
\text { - Unable to tolerate oral intake } \\
\text { - Severe vomiting unresponsive to antiemetics ( }>1 \text { day) } \\
\text { - Severe diarrhea unresponsive to antidiarrheal (>1 day) } \\
\text { - Uncontrolled pain or new severe pain } \\
\text { - Rash evaluation } \\
\text { - New or worsening extremity swelling } \\
\text { - Constipation unresponsive to bowel regimen } \\
\text { - Abnormal laboratory follow up } \\
\text { - Weight gain with need for diuresis } \\
\text { - Headache status post lumbar puncture } \\
\text { - Symptoms consistent with infection (cough, sore throat, dysuria, localized skin }\end{array}$ & $\begin{array}{l}\text { - Not receiving treatment with oncologist in the last } 3 \text { months } \\
\text { - Acute change in vital signs } \\
\text { - Respiratory rate }<10 \text { or }>30 \text { per minute } \\
\text { - Oxygen saturation }<92 \% \\
\text { - Heart rate }<50 \text { or }>120 \text { beats per minute } \\
\text { - Systolic blood pressure }<90 \text { or }>180 \mathrm{mmHg} \\
\text { - Acute oxygen requirement }>6 \mathrm{~L} \text { nasal cannula } \\
\text { - Requiring respiratory intervention (suctioning, etc.) } \\
\text { - Acute change in mental or neurologic status } \\
\text { - Acute onset of stroke-like symptoms } \\
\text { - New onset seizure } \\
\text { - Chest pain with suspected cardiopulmonary origin } \\
\text { - Symptomatic pericardial effusion } \\
\text { - Symptomatic dysrhythmia } \\
\text { - Cold pulseless extremity } \\
\text { - Pregnancy patients }\end{array}$ \\
\hline
\end{tabular}

group was similar (32.6\% ESI 2 and 64.2\% ESI 3). In contrast, the NCCHIC predominantly treated lower acuity patients $(65.8 \%$ calculated ESI of 4 or 5$)$. Of the 322 visits to the NCCHIC, 116 walked-in $(36.0 \%), 81$ presented after telephone triage $(25.2 \%)$, and 124 were referred by physicians $(38.5 \%)$. We were unable to determine referral origin for the remaining patient due to missing data.

The four most common chief complaints were the same in the ED groups pre- and post-intervention: pain, neurologic, fever/infection, and respiratory complaints. The four most common reasons for visit in the NCCHIC group were similar with one notable difference; neurologic complaints were replaced with gastrointestinal complaints.

\section{Discussion}

This report describes the population of patients with cancer seeking acute unscheduled care at a single academic site through a novel collaborative partnership between an ED and oncology clinic. Although no significant clinical difference exists in the demographic characteristics of ED patients and NCCHIC patients, differences do exist in the type of cancer included in the groups. The ED group appears to include more patients with solid tumors. The difference in type of cancer may reflect a lack of penetrance or familiarity with the new care pathway among different services-lines or inherent differences in the acuity of complications associated with the varying cancer types.
Notably, neurologic concerns were directed to the ED for evaluation per protocol. Consistent with this, patients with cancer of the CNS used the NCCHIC infrequently.

The reason for visit or chief complaint was similar in the ED and NCCHIC groups (pain, fever or infection, and respiratory complaint) except that the ED group also included neurologic complaints. This difference in presentation is once again reflective of the triage process that excludes neurologic complaints due to the need for rapid evaluation with advanced imaging.

Consistent with the developed triage model (Table 1), the ED group reflected higher acuity care $32.6 \%$ ESI 2 and $64.2 \%$ ESI 3 ) when compared to the NCCHIC group (65.8\% calculated ESI of 4 or 5$)$. This difference is also reflected in the over $10 \%$ admission rates of the postintervention ED group compared to the NCCHIC group (81.5\% versus $68.9 \%$ ). These admission rates are higher than admission rates from a national sample of cancer patients (57.2\%), suggesting differences in acuity, clinical decision-making, or the capacity for outpatient care between this study hospital and the more representative group of hospitals included in the CONCERN study [2, 12]. Although the ESI and admission rate are significantly different from the ED group, the persistently high admission rate in the NCCHIC group likely reflects the complexity of care required to address the supportive measures in this population. The high admission rates also likely enrichment due to the care model's referral pattern, where the patient's primary oncologist refers patients deemed 
Table 2 Patient demographics

\begin{tabular}{|c|c|c|c|c|}
\hline & & $\begin{array}{l}\text { Pre-intervention } \\
\text { emergency dept } \\
\mathrm{N}=1346 \\
\mathrm{n}(\%)\end{array}$ & $\begin{array}{l}\text { Post-intervention } \\
\text { emergency dept } \\
\mathrm{N}=1434 \\
\mathrm{n}(\%)\end{array}$ & $\begin{array}{l}\text { Post-interven- } \\
\text { tion NCCHIC } \\
\mathrm{N}=246 \\
\mathrm{n}(\%)\end{array}$ \\
\hline Age & Mean [IQR] & $61[50,73]$ & $62[51,73]$ & $61[51-69]$ \\
\hline Gender & Female & $764(56.8)$ & $791(55.2)$ & $110(44.7)$ \\
\hline \multirow[t]{3}{*}{ Race } & White & $780(57.9)$ & $872(60.8)$ & $158(64.2)$ \\
\hline & African American & $420(31.2)$ & $404(28.2)$ & $58(23.6)$ \\
\hline & Other/unknown & $146(10.8)$ & $158(11.0)$ & $30(12.2)$ \\
\hline \multirow[t]{3}{*}{ Ethnicity } & Hispanic & $81(6.0)$ & $90(6.3)$ & $15(6.1)$ \\
\hline & Not Hispanic & $1245(92.5)$ & $1320(92.1)$ & $227(92.3)$ \\
\hline & Unknown & $20(1.5)$ & $24(1.7)$ & $4(1.6)$ \\
\hline \multirow[t]{4}{*}{ Insurance } & Government & 780 (57.9) & $788(55.0)$ & $166(67.5)$ \\
\hline & Private & $555(41.2)$ & $505(35.2)$ & $56(22.8)$ \\
\hline & Other & $8(0.6)$ & $11(0.8)$ & $24(9.8)$ \\
\hline & Missing & $3(0.2)$ & $130(9.1)$ & $0(0)$ \\
\hline \multirow[t]{20}{*}{ Cancer type } & Central nervous system & $629(38.1)$ & 839 (46.7) & $2(0.8)$ \\
\hline & Gastrointestinal & $361(21.9)$ & $613(34.1)$ & $27(11.0)$ \\
\hline & Head and neck & $347(21.0)$ & $295(16.4)$ & $17(6.9)$ \\
\hline & Skin & $318(19.3)$ & $308(17.1)$ & $16(6.5)$ \\
\hline & Lung & $266(16.1)$ & $293(16.3)$ & $24(9.8)$ \\
\hline & Leukemia & $210(12.7)$ & $285(15.9)$ & $26(10.6)$ \\
\hline & Multiple myeloma & $121(7.3)$ & $103(5.7)$ & $22(8.9)$ \\
\hline & Gynecologic & $93(5.6)$ & $87(4.8)$ & $1(0.4)$ \\
\hline & Pancreatic & $106(6.4)$ & $120(6.7)$ & $8(3.3)$ \\
\hline & Lymphoma & $84(5.1)$ & $199(11.1)$ & $30(12.2)$ \\
\hline & Prostate & $69(4.2)$ & $52(2.9)$ & $8(3.3)$ \\
\hline & Bone & $43(2.6)$ & $59(3.3)$ & $5(2.0)$ \\
\hline & Bladder & $26(1.6)$ & $44(2.4)$ & $11(4.5)$ \\
\hline & Thyroid & $21(1.3)$ & $24(1.3)$ & $1(0.4)$ \\
\hline & Cancer NOS & $18(1.1)$ & $18(1.0)$ & $5(2.0)$ \\
\hline & Testicular & $15(0.9)$ & $46(2.6)$ & $3(1.2)$ \\
\hline & Breast & $11(0.7)$ & $89(5.0)$ & $24(9.8)$ \\
\hline & Renal & $10(0.6)$ & $1(0.1)$ & $13(5.3)$ \\
\hline & Myelodysplastic & $2(0.1)$ & $6(0.3)$ & $0(0)$ \\
\hline & Endocrine & $0(0)$ & $0(0)$ & $4(1.6)$ \\
\hline
\end{tabular}

NCCHIC North Carolina Cancer Hospital Infusion Center

beyond the scope of care provided in their clinic and requiring care in the NCCHIC or ED.

The number of ED visits in the post-intervention period increased when compared to the control group despite the initiation of the new care pathway and provision of 322 care encounters in the NCCHIC. This likely reflects the growing number of patients in care at the North Carolina Cancer Hospital and the increasing role the ED plays in caring for this patient population [1]. Among those NCCHIC visits, $76(23.6 \%)$ were repeat visits to the NCCHIC. The presence of repeat visits may indicate an acceptance by patients for this alternative site of care; however, further study is required as no patient satisfaction data was recorded. However, it is important to note that $51.4 \%$ of all ED visits in the post-intervention period occurred during NCCHIC hours of operations. Unfortunately, we are unable to comment on appropriateness of these visits to the ED for diversion to the NCCHIC, secondary to the retrospective nature of this study. Further refinement and implementation of this type of model for acute unscheduled cancer care may help reduce ED visits and over-crowding, particularly important during the current COVID-19 pandemic [15].

A significant proportion of patients experienced 30-ED return visits (18.5\% in the pre-ED group, $33.3 \%$ in the post-ED 
Table 3 Encounter characteristics

\begin{tabular}{|c|c|c|c|c|}
\hline & & $\begin{array}{l}\text { Pre-intervention emergency } \\
\text { dept. visits } \\
\mathrm{N}=1651 \\
\mathrm{n}(\%)\end{array}$ & $\begin{array}{l}\text { Post-intervention emergency } \\
\text { dept. visits } \\
\mathrm{N}=1797 \\
\mathrm{n}(\%)\end{array}$ & $\begin{array}{l}\text { Post-intervention } \\
\text { NCCHIC visits } \\
\mathrm{N}=322 \\
\mathrm{n}(\%)\end{array}$ \\
\hline \multirow[t]{5}{*}{ Disposition } & Admit & $1257(76.1)$ & $1465(81.5)$ & $222(68.9)$ \\
\hline & Discharge & $383(23.2)$ & $325(18.1)$ & $92(28.6)$ \\
\hline & Eloped/AMA/LWBS & $8(0.5)$ & $2(0.1)$ & $0(0)$ \\
\hline & Expired & $1(0.1)$ & $1(0.1)$ & $0(0)$ \\
\hline & Missing & $2(0.1)$ & $4(0.2)$ & $0(0)$ \\
\hline \multirow[t]{5}{*}{ Acuity (ESI) } & 1 & $20(1.2)$ & $15(0.8)$ & $0(0)$ \\
\hline & 2 & $505(30.6)$ & $586(32.6)$ & $42(13.0)$ \\
\hline & 3 & $1080(65.4)$ & $1153(64.2)$ & $68(21.1)$ \\
\hline & 4 & $40(2.4)$ & $38(2.1)$ & $180(55.0)$ \\
\hline & 5 & $2(0.1)$ & $0(0)$ & $32(9.9)$ \\
\hline ED return visit & Within 30 days & $306(18.5)$ & $599(33.3)$ & $142(44.1)$ \\
\hline Day of arrival & Weekday & $1256(76.1)$ & $1351(75.2)$ & $322(100)$ \\
\hline Time of arrival & Clinic open $(9 a-5 p)$ & $842(51.0)$ & $923(51.4)$ & $322(100)$ \\
\hline \multirow[t]{15}{*}{ Chief complaint } & Allergic & $3(0.2)$ & $7(0.4)$ & $6(1.9)$ \\
\hline & Bleeding & $99(6.0)$ & $105(5.8)$ & $6(1.9)$ \\
\hline & Blood clot & $14(0.8)$ & $9(0.5)$ & $5(1.6)$ \\
\hline & Cancer & $16(1.0)$ & $6(0.3)$ & $21(6.5)$ \\
\hline & Fever/infection & $163(9.9)$ & $196(10.9)$ & $67(20.8)$ \\
\hline & Gastrointestinal & $101(6.1)$ & $92(5.1)$ & $88(27.3)$ \\
\hline & Injury & $37(2.2)$ & $45(2.5)$ & $4(1.2)$ \\
\hline & Malaise & $23(1.4)$ & $22(1.2)$ & $19(5.9)$ \\
\hline & Neurologic & $222(13.4)$ & $252(14.0)$ & $15(4.7)$ \\
\hline & Pain & $513(31.1)$ & $656(36.5)$ & $41(12.7)$ \\
\hline & Psychiatric & $6(0.4)$ & $10(0.6)$ & $1(0.3)$ \\
\hline & Respiratory & $142(8.6)$ & $164(9.1)$ & $26(8.1)$ \\
\hline & Syncope & $75(4.5)$ & $71(4.0)$ & $8(2.5)$ \\
\hline & Other & $232(14.1)$ & $253(14.1)$ & $32(9.9)$ \\
\hline & Missing & $5(0.3)$ & $0(0)$ & $0(0)$ \\
\hline
\end{tabular}

$A M A$ against medical advice, $L W B S$ left without being seen, $E S I$ emergency severity index

NCCHIC North Carolina Cancer Hospital Infusion Center

group, and $44.1 \%$ in the NCCHIC group). These rates are consistent with return visit rates noted in the multi-center CONCERN study (26.6\%) [2]. These high proportions reflect the complexity of care these patients require and frequent need for acute unscheduled care. Due to the retrospective study design, we are unable to determine the factors affecting these return visits; however, increased adoption of this hybrid care model will help avoid inappropriate ED visits. Discharged ED patients represent an opportunity for NCCHIC treatment, as such further study of this population is required to inform future adaptation of this hybrid care model. Other research efforts including post-hospitalization transitions of care have demonstrated benefits in reducing subsequent care encounters and may help address the high rate of 30-day ED return visits [16].

\section{Limitations}

Although the data from this study provide insights into the population of patients with cancer seeking acute care, the findings are limited in part due to the use of a retrospective chart review study design. For example, ESI triage severity scores calculated retrospectively from chart review, as was done for the NCCHIC visits, may differ systematically from ESI scores determined by triage nurses during an ED visit. Characterization of primary cancer type for the ED dataset was not feasible; as a result, the classification system used may introduce systematic classification bias and limit informing care for specific cancer type subgroups. This may particularly affect the group with CNS involvement as this group includes both primary and metastatic CNS lesions. 
Although in the setting of acute care, the concern for devastating CNS complications in either situation is high and warrants aggressive evaluation justifying this population's exclusion from the NCCHIC. Additionally, this study was conducted at a single academic center affiliated with a comprehensive cancer center, which limits the generalizability of the study findings. However, this model of care could be replicated at any site where close collaboration between an oncology clinic and an ED is possible or co-located on the same campus. Important questions remain regarding the promptness with which care is provided, the quality of care, and patient satisfaction when using the combination of a cancer clinic plus the ED as compared to the ED alone; we did not collect data to answer these questions. These study results are still important as a proof of concept for a novel multidisciplinary acute care model for cancer patients seeking unscheduled medical care. Of note, this report reflects a period prior to the COVID-19 pandemic, which has significantly affected the provision of routine oncology care and emergency care [17-19]. Due to the current effect of the pandemic, all care models must be adapted to account for the required isolation of suspected COVID-19-infected patients and a clinic's ability to provide such care. This added challenge is best addressed by a multidisciplinary approach that best leverages the available resources of the various clinical care settings.

\section{Conclusion}

This report demonstrates the ability of a clinic and EDbased hybrid care model to address the acute care needs of patients with cancer. Results from this implementation study suggest that a novel partnership between the ED and an oncology clinic can serve as an alternate care location for low acuity patients.

Acknowledgements The authors would like to thank Sherri Morris, AGNP-BC, and Angela Nichols, AGNP-BC, for their support of this project and excelling patient care.

Author contribution All authors contributed to the study conception and design. Material preparation, data collection, and analysis were performed by JJB, MB, RBS, and TPM. The first draft of the manuscript was written by JJB and all authors commented on previous versions of the manuscript. All authors read and approved the final manuscript.

Funding Dr. Bischof was supported by the National Institutes of Health National Heart, Lung, and Blood Institute T32 training grant 2T32HL007149-41. The project described was supported by the National Center for Advancing Translational Sciences (NCATS), National Institutes of Health, through Grant Award Number UL1TR002489.
Data availability Not applicable.

Code availability Not applicable.

\section{Declarations}

Ethics approval This study was approved by the local institutional review board.

Consent to participate Not applicable.

Consent for publication Not applicable.

Conflict of interest MB, RBS report no conflict of interest. JJB has received speaker fees from the Cleveland Clinic foundation and the Association of Community Cancer Centers. FAC's institution receives support from Novartis, Merck, Replimune, and Amgen. A portion of her salary is derived from this support. TPM is employed by Quantworks, Inc. which has partnerships and contracts with companies doing work in the healthcare sector related to genetics, electronic health record utilization, biospecimen shipment, and symptom monitoring. TPM is also supported by contracts to support research funded by the $\mathrm{NIH}$, the Office of Research Integrity, and The John A. Hartford Foundation.

Disclaimer The content is solely the responsibility of the authors and does not necessarily represent the official views of the NIH.

\section{References}

1. Rivera DR, Gallicchio L, Brown J, Liu B, Kyriacou DN, Shelburne N (2017) Trends in adult cancer-related emergency department utilization: an analysis of data from the nationwide emergency department sample. JAMA Oncol 3:e172450

2. Caterino JM, Adler D, Durham DD, Yeung SJ, Hudson MF, Bastani A, Bernstein SL, Baugh CW, Coyne CJ, Grudzen CR, Henning DJ, Klotz A, Madsen TE, Pallin DJ, Reyes-Gibby CC, Rico JF, Ryan RJ, Shapiro NI, Swor R, Venkat A, Wilson J, Thomas CR Jr, Bischof JJ, Lyman GH (2019) Analysis of diagnoses, symptoms, medications, and admissions among patients with cancer presenting to emergency departments. JAMA Netw Open 2:e190979

3. Cooksley T, Marshall W, Ahn S, Lasserson DS, Marshall E, Rice TW, Klotz A (2020) Ambulatory emergency oncology: a key tenet of future emergencyoncology care. Int J Clin Pract 74(1):e13436

4. Young A, Marshall E, Krzyzanowska M, Robinson B, Brown S, Collinson F, Seligmann J, Abbas A, Rees A, Swinson D, Neville-Webbe H, Selby P (2016) Responding to acute care needs of patients with cancer: recent trends across continents. Oncologist 21:301-307

5. Ahn S, Lee YS, Lim KS, Lee JL (2012) Emergency department cancer unit and management of oncologic emergencies: experience in Asan Medical Center. Support Care Cancer 20:2205-2210

6. Cooksley T, Campbell G, Al-Sayed T, LaMola L, Berman R (2018) A novel approach to improving ambulatory outpatient management of low risk febrile neutropenia: an Enhanced Supportive Care (ESC) clinic. Support Care Cancer 26:2937-2940

7. Delatore LR (2018) The Cancer Emergency Department-The Ohio State University James Cancer Center experience. Emerg Med Clin North Am 36:631-636 
8. Handley NR, Schuchter LM, Bekelman JE (2018) Best practices for reducing unplanned acute care for patients with cancer. J Oncol Pract 14:306-313

9. Mazur KA, McCarthy K, Suell MN, Hockenberry MJ (2012) An outpatient Urgent Care Bay within a pediatric cancer and hematology center. J Pediatr Oncol Nurs 29:206-210

10. Ruegg TA (2013) A nurse practitioner-led urgent care center: meeting the needs of the patient with cancer. Clin J Oncol Nurs 17:E52-57

11. Mayer DK, Travers D, Wyss A, Leak A, Waller A (2011) Why do patients with cancer visit emergency departments? Results of a 2008 population study in North Carolina. J Clin Oncol 29:2683-2688

12. Adler D, Abar B, Durham DD, Bastani A, Bernstein SL, Baugh CW, Bischof JJ, Coyne CJ, Grudzen CR, Henning DJ, Hudson MF, Klotz A, Lyman GH, Madsen TE, Pallin DJ, Reyes-Gibby CC, Rico JF, Ryan RJ, Shapiro NI, Swor R, Thomas CR Jr, Venkat A, Wilson J, Yeung SJ, Caterino JM (2019) Validation of the emergency severity index (version 4) for the triage of adult emergency department patients with active cancer. J Emerg Med 57:354-361

13. Gilboy N, Tanabe P, Travers DA, Rosenau AM (2011) Emergency severity index (ESI): a triage tool for emergency department care Version 4 Implementation Handbook 2012 edition. Agency for Healthcare Research and Quality (AHRQ Publication No. 12-0014)

14. Harris PA, Taylor R, Thielke R, Payne J, Gonzalez N, Conde JG (2009) Research electronic data capture (REDCap)-a metadatadriven methodology and workflow process for providing translational research informatics support. J Biomed Inform 42:377-381
15. Cooksley T, Font C, Scotte F, Escalante C, Johnson L, Anderson R, Rapoport B (2021) Emerging challenges in the evaluation of fever in cancer patients at risk of febrile neutropenia in the era of COVID-19: a MASCC position paper. Support Care Cancer 29:1129-1138

16. Hanson LC, Collichio F, Bernard SA, Wood WA, Milowsky M, Burgess E, Creedle CJ, Cheek S, Chang L, Chera B, Fox A, Lin FC (2017) Integrating palliative and oncology care for patients with advanced cancer: a quality improvement intervention. J Palliat Med 20:1366-1371

17. Hartnett KP, Kite-Powell A, DeVies J, Coletta MA, Boehmer TK, Adjemian J, Gundlapalli AV (2020) Impact of the COVID-19 pandemic on emergency department visits - United States, January 1, 2019-May 30, 2020. MMWR Morb Mortal Wkly Rep 69:699-704

18. Jazieh AR, Akbulut H, Curigliano G, Rogado A, Alsharm AA, Razis ED, Mula-Hussain L, Errihani H, Khattak A, De Guzman RB, Mathias C, Alkaiyat MOF, Jradi H, Rolfo C (2020) Impact of the COVID-19 pandemic on cancer care: a global collaborative study. JCO Glob Oncol 6:1428-1438

19. Richards M, Anderson M, Carter P, Ebert BL, Mossialos E (2020) The impact of the COVID-19 pandemic on cancer care. Nat Cancer 20:1-3

Publisher's note Springer Nature remains neutral with regard to jurisdictional claims in published maps and institutional affiliations.

Presentations A portion of this work was presented as a poster presentation at the 2020 Oncologic Emergency Medicine Conference, Houston, TX. 